\title{
Prevalência das hepatites B e C em moradores de rua em São Luís-MA
}

\author{
Prevalence of hepatitis $B$ and $C$ in a hundred homeless people in the city of São Luis - MA
}

Thiago Henrique dos Santos Silva ${ }^{1}$, Mariana Martins Calisto ${ }^{2}$, Ana Carolina Macedo de Carvalho², Hilário José Cardoso Magalhães², Valério Monteiro-Neto ${ }^{1,3}$, Enzo Eldes Costa Ribeiro', Mariana Maryelle Ferreira de Sousa ${ }^{1}$, Domingos Magno Santos Pereira ${ }^{1}$, Silvio Gomes Monteiro ${ }^{1,3}$

\begin{abstract}
Resumo: Prevalência de hepatite $\mathrm{B}$ e $\mathrm{C}$ e identificação do perfil epidemiológico de cem moradores de rua de São Luís-Maranhão. Objetivos: Caracterizar o perfil epidemiológico dos indivíduos portadores dos vírus das hepatites B e C; Analisar a prevalência e vulnerabilidade a ISTs em moradores de rua em São LuísMA;. Métodos: Trata-se de um estudo epidemiológico do tipo transversal, descritivo-analítico de população de risco, ou seja, de moradores das ruas. O projeto foi aprovado pelo Comitê de Ética em Pesquisa da Universidade Ceuma com seguinte $n^{\circ}$ de parecer: 769.163. Resultados:. Na amostra com 105 moradores de rua de São Luis encontrou-se as seguintes prevalências: Hepatite $C(3,8 \%)$ e não foi encontrado dentro do espaço amostral nenhum caso de hepatite B. Consumo de drogas: Crack $(21,9 \%)$, Crack e Cocaína(40,0\%), Cocaína(19,0\%), Maconha(12,4\%), Ignorado(6,7\%). População composta em sua maioria $(53,3 \%)$ por homens, (100\%) heterossexuais, $(59,5 \%)$ não brancos. Conclusão: os dados obtidos demonstraram uma grande vulnerabilidade desse grupo populacional quanto as ISTs o que torna preocupante e de extrema importância a assistência a essa parcela da população de São Luis tanto no esclarecimento das medidas preventivas das ISTs quanto na deteç̧ão precoce dessas doenças que podem se manifestar de forma silenciosa tais como a Hepatite B e C impactando assim na qualidade de vida desse grupo negligenciado pelas políticas públicas.
\end{abstract}

Palavras-Chave: Hepatite B; Hepatite C; Moradores de Rua; IST; Prevalência;

\begin{abstract}
Prevalence of hepatitis $B$ and $C$ and selection of the epidemiological profile of a hundred homeless people in São Luís-Maranhão. Objectives: To characterize the epidemiological profile of individuals with hepatitis B and C; To analyze the prevalence and vulnerability to STIs in homeless people in São Luís- MA; METHODS: This is an epidemiological cross-sectional, descriptive-analytical study of at-risk population, that is, street dwellers. The project was approved by the Research Ethics Committee of the Ceuma University with the following number: 769.163. Results :. The following prevalence's were found in the sample with a hundred and five homeless: Hepatitis C $(3.8 \%)$ were found, and no positive cases of hepatitis B. Drug use: Crack (21.9\%), Crack and Cocaine (40.0\%), Cocaine (19.0\%), Marijuana (12.4\%), Ignored $(6.7 \%)$. Population composed mostly $(53,3 \%)$ by men, $(100 \%)$ heterosexual, nonwhite $(59,5 \%)$. Conclusion: The data showed a high vulnerability of this population group to STIs, which makes it very worrying and extremely important to care about this non assisted population of São Luis, in order to clarify the preventive measures of STIs and in the early detection of these diseases. such as Hepatitis B and C, thus impacting the quality of life of this group neglected by public policies
\end{abstract}

Keywords: Hepatitis B; Hepatitis C; Street dwellers; IST; Prevalence;

\footnotetext{
${ }_{1}^{1}$ Programa de Pós-Graduação em Biologia Parasitária, Universidade Ceuma, São Luis-MA, Brazil

${ }^{2}$ Coordenação de Medicina, Universidade Ceuma, São Luis-MA, Brazil

${ }^{3}$ Centro de Ciências Biológicas e da Saúde, Universidade Federal do Maranhão, São Luis-MA, Brazil

${ }^{*}$ Autor correspondente - Prof. Dr. Silvio Gomes Monteiro

Rua Josué Montello no1, Renascença II - São Luís-MA. CEP 65.075-120. Tel.: (98) 32141427
} 


\section{Introdução}

As IST's por serem doenças de longa trajetória na vida humana, aparecem frequentemente em culturas unilaterais, direcionadas em função das atividades sexuais. $O$ aumento dessas doenças vem ocorrendo em virtude de mudanças no comportamento sexual que ocorrem predominantemente na população mais jovem, sendo estes responsáveis atualmente por uma grande totalidade dos casos de infecções via transmissão sexual. ${ }^{1}$

Os adolescentes iniciam cedo sua vida sexual, tomam iniciativas incontroladas, muitas vezes motivadas por curiosidade e sem nenhuma informação quanto a formas de prevenção, contribuindo, portanto, para a disseminação das ISTs. Nesse sentido, o processo de descoberta da sexualidade quando associado à iniciação precoce da atividade sexual, tem como conseqüências 0 surgimento de comportamentos de risco, como o ato desprotegido, motivado em algumas ocasiões pelo uso abusivo de substancias de teor alcoólico, tabagismo, ou até mesmo o uso de drogas ilícitas, aumentando a exposição desses jovens às infecções sexualmente transmissíveis/HIV. ${ }^{2}$

A epidemia de AIDS no Brasil é um grande problema de saúde entre a população. ${ }^{1}$ As ISTs só voltaram a readquirir importância como problema de saúde pública após a epidemia da AIDS. Estudos demonstraram que pessoas portadoras de ISTs e infecções genitais não ulcerativas têm risco aumentado de cinco a 10 vezes de se infectar pelo HIV, o que sobe para 18 vezes se a doença cursa com lesões ulceradas. ${ }^{3}$

As IST's são causadas por microorganismos patogênicos (bactérias, vírus ou fungos) que se inserem no interior do corpo do homem e da mulher, usualmente através dos órgãos genitais. Estas doenças não escolhem idade, classe social, raça ou condição financeira. Qualquer um pode se contaminar, sendo a prevenção a melhor atitude a ser tomada. A contaminação ocorre principalmente pelo contato sexual com um (a) parceiro (a) contaminado (a), independentemente da relação ser efetuada entre indivíduos do mesmo sexo ou de sexos opostos. ${ }^{1}$

São muitos os caminhos que levam um jovem a ter relações sexuais desprotegidas, dentre eles encontra-se o próprio desconhecimento dos mecanismos dessas doenças e formas de prevenção. Além disso, os números que vem a tona sobre as ISTs, sem dúvidas, são menores do que os números reais. Em virtude da dificuldade de obtê-los e do grande número de ISTs existentes. ${ }^{4}$

Nesse sentido, os moradores de rua também podem ser considerados um público que se encontra exposto a fatores de risco contínuos para a contaminação de ISTs. Isso se da principalmente ao comportamento sexual e ao uso de drogas injetáveis. ${ }^{5}$

Dessa forma, em grupos populacionais com índice elevado de vulnerabilidade, é importante a formação de um planejamento de ação estratégico. De modo que demande o reconhecimento de aspectos socioeconômicos e demográficos, visando estabelecer um perfil epidemiológico das pessoas portadoras ou que se apresentam em situações de risco para o possível surgimento das IST's. 6,7

\section{Material e Métodos}

Trata-se de um estudo epidemiológico do tipo transversal, descritivo-analítico de população de risco, ou seja, de moradores de rua. O projeto e o termo de consentimento livre e esclarecido aprovados pelo Comitê de Ética em Pesquisa da Universidade Ceuma com o seguinte número de parecer: 769.163 .

O cálculo amostral dos moradores de rua foi feito através do programa 
estatístico PASS (Power Analysis \& Sample Size) 11 (2011). Para o cálculo do tamanho amostral dos grupos dos moradores de rua, foi padronizado conforme os seguintes parâmetros: $39,6 \%$ da prevalência de alguma IST em moradores de rua ${ }^{8}$, erro tolerável de $7 \%$, nível a de $5 \%$, poder do teste de $80 \%$, mais $5 \%$ de possíveis perdas, sendo o número mínimo de moradores de rua de 86.

A coleta dos dados dos moradores de rua foi realizada de maneira randomizada em ruas e praças do centro de São Luis, próximo aos locais onde realizam suas refeições e em locais de assistência a este público, durante 0 período de maio de 2014 a outubro de 2014. Os participantes da pesquisa foram abordados e esclarecidos sobre os objetivos da pesquisa, os riscos e os benefícios, além de serem solicitados a assinar o Termo de Consentimento Livre e Esclarecido (TCLE) caso concordassem em participar da pesquisa. Aceitando participar da pesquisa, houve o preenchimento de forma voluntária e espontânea de um questionário estruturado sobre as ISTs (Infecções Sexualmente Transmissíveis). E em seguida realizada foi feita a coleta com uma amostra digital de sangue dos participantes para a utilização do kit de testes rápidos do diagnóstico para hepatites $\mathrm{B}$ e $\mathrm{C}$. A amostra de sangue foi devidamente coletada por profissionais de saúde equipados e qualificados.

O instrumento utilizado foi através de um questionário contendo questões fechadas e abertas com a finalidade de:

a) Caracterizar a população a ser estudada;

b) Conhecer aspectos do cotidiano pessoal e profissional;

c) Conhecer o comportamento sexual;

d) Identificar o nível de conhecimento sobre as IST/AIDS;

Os dados foram avaliados pelo programa IBM SPSS Statistics 20 (2011). Inicialmente, sendo realizadas as análises da estatística descritiva, ou seja, gráficos e tabelas de freqüência das variáveis analisadas e estimar a média, desvio-padrão, máximo e mínimo das variáveis numéricas

\section{Resultados}

Em todo o estudo conseguiu-se avaliar 105 moradores de rua de São Luís, no período de maio de 2014 a outubro de 2014. A pesquisa não apresentou nenhuma desistência. Aferiuse que entre os entrevistados, $49(46,7 \%)$ eram do sexo feminino e 56 (53,3\%) eram do sexo masculino.

No que diz respeito a aspectos sócio-demográficos dos participantes, 42 (40\%) se autoreferiram como da raça caucásia ("branca"), 31 (29,5\%) da raça parda e 32 (30,5\%) da raça negra. Possuíam uma média de idade de 35,3 \pm 9,2 anos, sendo apenas quatro participantes $(3,8 \%)$ com menos de 21 anos e $6(5,7 \%)$ com idade igual ou superior a 50 anos.

A renda mensal média foi de $R \$$ $119,0 \pm 64,6$ reais, sendo a fonte de renda principal referente à esmola para 60 entrevistados $(57,1 \%)$. Ao analisar a procedência, foi visto que 97 (92,38\%) eram procedentes de São Luís - MA e 8 $(7 \%)$ de outros municípios, predominantemente do interior do estado do Maranhão.

Quanto ao estado civil dos mesmos, $64(61 \%)$ declararam-se solteiros, 22 (21\%) casados, 4 (3,8\%) em união estável, $15 \quad(14,3 \%) \quad$ separados/ divorciados.

Ainda avaliando as variáveis sóciodemográficas, foi visto que $37(35,2 \%)$ eram analfabetos, 49 (46,3\%) com o ensino fundamental completo e 19 $(18,1 \%)$ com o ensino médio completo. A duração média em anos de vivência nas ruas foi de 13,4 \pm 9,9 anos.

Ao analisar 0 perfil de comportamento sexual, 105 (100\%) dos participantes declararam-se heterossexuais. Destes, $66 \quad(62,9 \%)$ 
afirmaram procurar por parceiro (a) nas ruas, enquanto $39(37,1 \%)$ afirmaram não procurar por parceiro (a) nas ruas. Dentre os principais motivos envolvidos nos aspectos motivacionais relacionados à busca dos parceiros (as) nas ruas, houve uma predominância no que diz respeito à carência e a necessidade, assumindo valores de $40 \quad(60,6 \%)$ participantes sendo motivados prioritariamente por carência e $24(36,4 \%)$ por necessidade. Aqueles que referiram não buscar parceiro (a) nas ruas relataram que 0 principal motivo da negação era motivado por medo de contágio, sendo estes 39 $(100 \%)$ dos participantes. Dos que relataram realizar relações sexuais nas ruas, $14(21,2 \%)$ negam 0 uso de preservativo e $49(74,2 \%)$ afirmam o uso da proteção. Dentre os que usam o preservativo $49(100 \%)$ utilizam como forma de prevenção de doenças. Assumindo grande maioria dos entrevistados, $74(70,5 \%)$ dos participantes declararam nunca ter tido nenhum tipo de infecção sexualmente transmissível, em contrapartida 31 $(29,5 \%)$ declararam já ter sido portador de alguma IST.

Em relação às Infecções Sexualmente Transmissíveis apresentadas durante a pesquisa, considerando todos os testes, a prevalência geral de ISTs foi de 17 $(16,2 \%)$ participantes, apresentando 88 $(83,8 \%)$ de casos negativos. Dentre as doenças encontrou-se a seguinte prevalência: 4 ( 3,8\%) dos casos positivos para HIV, bem como $4(3,8 \%)$ para Hepatite C, 11 (10,5\%) positivos para Sífilis, e nenhum caso positivo para Hepatite B. Dos entrevistados, 31 (29,\%) afirmavam já ter tido alguma IST confirmada antes da realização da pesquisa. Dentre os que apresentaram IST previamente, $19(61,3 \%)$ relataram já ter tido Gonorreia, 10 (32,3\%) Herpes e 2 $(6,5 \%)$ dos participantes, sífilis.

Em relação a hábitos de vida $\mathrm{e}$ outras características que estabelecem 0 perfil epidemiológico dos entrevistados,
$10(9,5 \%)$ relataram ser usuários de drogas injetáveis, $72(68,6 \%)$ tinham tatuagem e $47 \quad(44,8 \%)$ disseram compartilhar itens de higiene pessoal com outras pessoas. $80(76,2 \%)$ dentre os participantes relataram ser etilistas e $70(66,7 \%)$ tabagistas. $85(81,0 \%)$ já fizeram uso de drogas ilícitas. Dentre elas: Crack 23 (21,9\%), Crack e Cocaína concomitantemente $42(40,0 \%)$, Cocaína $20(19,0 \%)$, Maconha $13(12,4 \%)$ dos casos.

No que diz respeito a outros aspectos relacionados à saúde 34 $(32,4 \%)$ dos participantes referiram que já sentiram algum tipo de dor no peito. 7 $(6,7 \%)$ já apresentaram infarto agudo do miocárdio (IAM), 29 (27,6\%) referiram ser portadores de dor lombar e apenas 1 (1\%) participante já apresentou sintomas correspondentes a insônia.

\section{Discussão}

A pesquisa englobou 105 moradores de rua sendo a primeira pesquisa realizada com esse público na região de São Luís do Maranhão. No Brasil, a população em situação de rua, encontra-se em um alarmante nível de exclusão social e em sua maioria convivem com a pobreza, além do baixo padrão de higiene pessoal e ambiental. Esses fatores fazem com que os mesmos encontrem-se sujeitos a doença física e mental, isolamento social e político. ${ }^{8}$

Observou-se que dentre os entrevistados, 56 (53,3\%) eram do sexo masculino e $49(46,7 \%)$ do sexo feminino. Havendo uma discreta prevalência no número de moradores de rua do sexo masculino ao feminino, conforme outras pesquisas demonstradas na literatura ${ }^{9}$

No presente estudo, 64 (61,0\%) eram solteiros (as) e a idade média dos participantes era de 35 anos. Correspondendo com que é encontrado em pesquisas realizadas com moradores de rua que tem o intuito de avaliar a 
correlação dos mesmos com a alta prevalência de ISTs. ${ }^{9-11}$

O uso de drogas intravenosas (DIV) é uma das principais formas de transmissão do HCV nos últimos 40 anos em países como os Estados Unidos e a Austrália, e atualmente este é o principal fator de risco em países desenvolvidos. Nesses países, o uso de DIV responde por cerca de $70 \%$ a $80 \%$ das contaminações pelo HCV ocorridas nos últimos 30 anos. ${ }^{12-15}$

No Brasil, a principal forma de contaminação também é através do uso de drogas injetáveis, considerando-se ainda a transfusão sanguínea como um fator de risco igualmente alto, segundo dados epidemiológicos ${ }^{16}$ Tendo em vista que a via hematogênica, é uma das mais prevalentes formas de contaminação de ISTs, em especial a Hepatite Viral $\mathrm{C}^{17}$ dos entrevistados, 10(9,5\%) alegaram uso das mesmas e estiveram, portanto, expostos ao risco de contaminação. Por outro lado 105 (100\%) dos participantes da pesquisa negam realização de transfusão sanguínea prévia. Dentre os resultados encontrados, os testes rápidos detectaram $4(3,8 \%)$ casos positivos para HVC no espaço amostral exposto de 105 moradores de rua.

As taxas de detecção de hepatite $B$ no Brasil, desde 2011, vêm apresentando poucas variações, com leve tendência de queda desde 2014, atingindo 6,5 casos para cada 100 mil habitantes no país em $2017 .{ }^{18}$ No entanto, algumas variáveis encontram-se correlacionadas com a incidência da Hepatite $B$ no país. Conforme o estudo ${ }^{19}$, o baixo índice de escolaridade demonstra alto fator de risco para contaminação por Hepatite Viral B. No entanto, no presente estudo não houve casos detectados de Hepatite B, apesar de apresentar $37 \quad(35,2 \%)$ analfabetos e 49 (46,7\%) participantes que alegaram somente 0 ensino fundamental incompleto.

Observa-se que, dos 30 participantes que admitiram ter alguma doença sexualmente transmissível previamente à realização da pesquisa, houve uma incidência de 19 (61,3\%) casos por Gonorreia, seguido de 10 $(32,3 \%)$ casos de Herpes Vírus. Condizendo fortemente com a literatura, no que diz respeito à elevada prevalência dessas mesmas doenças entre moradores de rua da cidade de São Paulo. ${ }^{9}$

O trabalho avaliou ainda, a precariedade das condições de vida dos moradores de rua da cidade de São Luís - MA e a possível associação com o aparecimento de ISTs. Tendo em média duas refeições diárias, e uma renda mensal média dos participantes da pesquisa no valor $R \$ 119,0$ reais, adquiridos através de esmolas ou através da vigilância de carros, esse valor não corresponde a um salário mínimo. O Maranhão encontra-se no cenário nacional como uma das unidades da federação com os piores indicadores sociais. Da população de 6,5 milhões de habitantes, 1,7 milhão está abaixo da linha da miséria, corroborando diretamente com o que há de descrito na literatura quanto a relação dos níveis socioeconômicos e sociais precários associados intimamente aos portadores de hepatites virais. ${ }^{20,21}$

Dentre os entrevistados, 66 (62,9\%) assumiram ter relações sexuais com parceiro (a) morador de rua, e desses, 49 $(74,2 \%)$ afirmaram usar camisinha como forma de proteção, o que sugere que a população estudada tem certo nível de conhecimento no que diz respeito à eficácia deste método de prevenção.

O uso de tatuagem é uma variável relacionada intimamente ao aumento do risco de contágio de infecções sexualmente transmissíveis. ${ }^{22} \mathrm{Na}$ presente pesquisa, a maioria dos moradores de rua tinha tatuagem, um total de 72 (68,6\%), aumentando sua exposição ao risco.

Conforme estudo, Entre 210 entrevistados que responderam à questão sobre compartilhamento de equipamentos de higiene, como escovas 
de dente e barbeadores, 89 responderam afirmativamente. ${ }^{9}$ Ou seja, o equivalente a $42 \%$, semelhante ao percentual médio obtido no presente estudo, onde em um espaço amostral de 150 moradores de rua, 47 (44,8\%) alegaram compartilhamento de itens de higiene pessoal.

No que diz respeito a participação prévia dos participantes em palestras, observou-se que $60 \quad(57,1 \%)$ dos entrevistados nunca presenciaram a palestras que abordassem 0 tema envolvendo medidas de prevenção e 74 $(75,5 \%)$ dos participantes não tem conhecimento sobre nenhuma iniciativa do governo voltada para a conscientização e prevenção adequada quanto ás ISTs. Entretanto, apesar da baixa acessibilidade, os $49(74,2 \%)$ participantes dentre os $66(62,9 \%)$ que disseram manter relações sexuais nas ruas, admitiram utilizar preservativos e referem que o principal motivo para o uso é quanto à proteção. Contudo, 55\% dos entrevistados admitiram acreditar que 0 principal motivo da contaminação por ISTs entre os moradores de rua era atribuído ao uso de drogas injetáveis e seus compartilhamentos. Em decorrência desses fatos, não se exclui a real necessidade de políticas públicas voltadas para a instrução dos moradores de rua quanto aos possíveis meios existentes de contaminação e prevenção ao contágio por Infecções Sexualmente Transmissíveis.

\section{Conclusão}

A pesquisa realizada evidenciou que grande parte dos moradores de rua de São Luís são procedentes da capital e regiões do interior do estado e que vivem em condições socioeconômicas muito precárias. Com 'índice de analfabetismo muito alto e com diversas patologias seja de origem psicossomática ou orgânica.

O uso do álcool, tabaco e drogas ilícitas, principalmente crack e cocaína é elevado. Já o uso das drogas injetáveis a frequência é baixa, mesmo assim é mais uma vulnerabilidade dentro desse grupo.

Dentre as ISTs mais prevalentes nos moradores de rua, observou-se que gonorreia era a doença mais frequente, buscando-se posteriormente atendimento terapêutico em farmácias.

Cabe ressaltar que, apesar de não serem identificados índices numericamente relevantes relacionados ao surgimento de hepatite $B$ e $C$ entre os moradores de rua da atual pesquisa, foi observado que esse público se encontra permanentemente exposto a fatores de risco confirmados na literatura. $O$ que denota a real necessidade de políticas públicas voltadas para a informação e assistência das pessoas em situações de rua.

Quanto ao comportamento sexual, todos os entrevistados afirmaram ser heterossexuais. A grande maioria admite procurar por parceiro (a) nas ruas e usar preservativo durante as relações sexuais. O principal motivo que os motiva ao uso de preservativos é a prevenção de doenças. Entretanto, uma certa parcela da amostra afirmou não usar camisinha com as parceiras fixas. Esse fato denota a existência de conhecimento por parte dos moradores de rua, no que diz respeito a principal via de disseminação de Infecções Sexualmente Transmissíveis.

Contudo, considerando a alta incidência de doenças transmitidas predominantemente por via sexual entre a população em situação de rua, e analisando ainda, a falta de acessibilidade desse mesmo público ás políticas pública de assistência a saúde e educação sexual, é de suma importância a formulação de medidas pelos órgãos públicos. Essas devem ser voltadas para a orientação dos moradores de rua quanto a formas de contágio e medidas profiláticas que previnem o surgimento de ISTs, em especial as Hepatites virais B e $C$ que são doenças que se manifestam silenciosamente e possuem consequências graves aos portadores, 
além de exigirem altos investimentos para a saúde pública.

\section{Agradecimento}

Esta pesquisa foi financiada pela Fundação de Amparo de Pesquisa e Desenvolvimento do Estado do maranhão (FAPEMA).

\section{Referências}

1. Brasil, Departamento de DST/HIV/AIDS e hepatites virais. Boletim Epidemiológico AIDS e DST. Ano 8.1 (2011) p.162

2. De Moraes, Silvia Piedade, and Maria Sylvia de Souza Vitalle. Direitos sexuais e reprodutivos na adolescência. Revista da Associação Médica Brasileira 58.1 (2012): 48-52.

3. Fleming, Douglas T., and Judith $\mathrm{N}$. Wasserheit. From epidemiological synergy to public health policy and practice: the contribution of other sexually transmitted diseases to sexual transmission of HIV infection. Sexually Transmitted Infections 75.1 (1999): 3-17.

4. Neves, Rosália Garcia, et al. Simultaneidade de comportamentos de risco para infecções sexualmente transmissíveis em adolescentes brasileiros, 2012. Epidemiologia e Serviços de Saúde 26 (2017): 443-454.

5. Robertson, Marjorie J., et al. HIV seroprevalence among homeless and marginally housed adults in San Francisco. American Journal of Public Health 94.7 (2004): 1207-1217.

6. Custódio, Geisiane, et al. Comportamento sexual e de risco para DST e gravidez em adolescentes. DST J Bras Doenças Sex Transm 21.2 (2009): 60-4.

7. Pereira, Bianca de Souza, et al. Fatores associados à infecção pelo HIV/AIDS entre adolescentes e adultos jovens matriculados em Centro de Testagem e Aconselhamento no Estado da Bahia, Brasil. Ciência \& Saúde Coletiva 19 (2014): 747-758.
8. Grangeiro, Alexandre, et al. Prevalence and vulnerability of homeless people to HIV infection in São Paulo, Brazil. Revista de Saude Publica 46.4 (2012): 674-684.

9. Brito, Valquiria OC, et al. Infecção pelo HIV, hepatites B e C e sífilis em moradores de rua, São Paulo. Revista de Saúde Pública 41 (2007): 47-56.

10. Deienno, Marylei Castaldelli Verri, et al. Perfil dos usuários do serviço de aconselhamento no serviço de assistência especializada em DST/Aids Campos Elíseos, município de São Paulo, Brasil. BEPA. Boletim Epidemiológico Paulista (Online) 7.74 (2010): 13-22.

11. Bassichetto, Kátia Cristina, et al. Epidemiological profile of HIV positive individuals from a HIV/STD Counseling and Testing Center (CTA) in the city of São Paulo, Brazil.Revista Brasileira de Epidemiologia 7.3 (2004): 302-310.

12. Alter, Miriam J. Prevention of spread of hepatitis C. Hepatology 36.S1 (2002): S93S98.

13. Dore, Gregory J., et al. Epidemiology of hepatitis $C$ virus infection in Australia. Journal of Clinical Virology 26.2 (2003): 171-184

14. Hagan, Liesl M., and Raymond F. Schinazi. Best strategies for global HCV eradication. Liver International 33 (2013): 68-79.

15. Ly, Kathleen N., et al. The increasing burden of mortality from viral hepatitis in the United States between 1999 and 2007. Annals of Internal Medicine 156.4 (2012): 271-278.

16. Brasil, A B C D E de Hepatites para comunicadores, Brasília: Secretaria de Vigilância em Saúde/MS. (2005) p.14

17. Bittencourt, Greicy Kelly Gouveia Dias, et al. Beliefs of older adults about their vulnerability to HIV/Aids, for the construction of nursing diagnoses. Revista Brasileira de Enfermagem 68.4 (2015): 579-585

18. BrasilL, Brasília: "Secretaria de Vigilância em Saúde/MS. Boletim Epidemiológico Volume 49 (2018) No 31

19. Dias, Jerusa Araújo, Crispim Cerutti Júnior, and Aloísio Falqueto. Fatores associados à infecção pelo vírus da hepatite B: um estudo caso-controle no município de São Mateus, Espírito Santo. Epidemiologia e Serviços de Saúde 23 (2014): 683-690.

20. Censo demográfico 2010. Disponivel em : http://www.ibge.gov.br

21. Teles, Sheila Araújo. "Hepatites Virais: um desafio para enfermagem. Revista Brasileira de Enfermagem 70.2 (2017): 243-244. 
22. Carvalho, Paulie Marcelly Ribeiro dos Santos, et al. Prevalence of signs and symptoms and knowledge about sexually transmitted diseases. Acta Paulista de Enfermagem 28.1 (2015): 95-100. 